\title{
Sign Language Interpreter: What Makes it Different?
}

\author{
Mr. Abdelaziz Arssi Phd Candidate, Pr. Nour Taibi \\ University Mohammed V, Faculty of Education, Rabat, Morocco
}

\begin{abstract}
Understanding the situation of sign language (SL) and its history is essential to comprehend the role of sign language interpreter better. It has often implied fundamental questions that refer to the identity construction of the person, his/her attachment to a linguistic community and the place of this community in the current social scheme. Sign language is like any other human languages; it spontaneously develops through deaf communities using this language and without external interference from hearing persons. All living human languages, whether spoken or signed, are characterised by the multiplicity, diversity, and the evolving nature of their vocabulary and this is what determines and proves of the richness of a language. Those who interpret from spoken to sign language are usually referred to as "sign language interpreters" and "deaf interpreters", but both terms are unsatisfactory because both apply only to the language or community to which they serve. This article will try to shed light on the origins of sign language as well as the emergence of sign language interpreters as key players in an everlasting debate round the deaf and the deaf rights throughout nearly three centuries.
\end{abstract}

Keywords: sign language (SL), deaf, bilingual education, interpreting, communication

\section{Introduction}

Sign language interpreters have always been critical elements in the life of deaf people all over the world as they allow them to be more and more autonomous. Before, deaf people depended on the benevolence of their families to help them communicate. However, despite all this goodwill, parents, brothers, and sisters were not professionals. If they manage to interact with relatives, they do not all have sufficient command of spoken languages to communicate in depth with unknown third parties, even if only in writing. The use of professional sign language interpretation has become more frequent and has also become a persistent claim to be practised in a wide variety of fields. The role of the interpreter is, therefore, both essential and complicated.

Unlike many interpreters in spoken languages, sign interpreters work collaboratively with deaf people. The growing demand for quality SL interpreting for the deaf attracted the attention of researchers (Ressler, 1998; Cerney, 2004) who focused on some aspects of the interpreting process occurring in SL interpreting during a staged lecture in spoken languages and its subsequent interpretation into SL. Studies examined the correspondence of the message conveyed from the interpreter to the deaf audience. There is substantial evidence that in the relevant cognitive processes that interpreters working between the SL and the spoken language, for

Mr. Arssi Abdelaziz, MA, Communication and Translation, Department of English, Faculty of Education, University Mohammed V, Rabat, Morocco.

Pr. Nour Taibi, PhD, Applied Linguistics, Department of English, Faculty of Education, University Mohammed V, Rabat, Morocco. 
example, do the same job, but differently from interpreters who deal with spoken languages (Isham \& Lane, 1993). The recognition of sign language as an official language of the deaf in different countries has meant that those countries (Sweden, USA, Australia, Canada, and France) had to provide SL interpreters in conferences. In particular, those dealing with deafness in all its aspects (linguistic, cognitive, social, or cultural), meetings to which the deaf were willing to assist, especially the small fraction of them who have become professionals of deafness, particularly teachers of sign language, even educators, and in minimal numbers, technical teachers or general education.

\section{Deaf Community}

Deaf community according to the World Health Organisation (WHO) global estimates in 2017, over five per cent (360 million people) of the world's population have disabling hearing loss including 328 million adults and 32 million children. Approximately $15 \%$ of the world's adult population has some degree of hearing loss and $25 \%$ of them are aged above 65 years. A global survey conducted by the World Federation of the Deaf (WFD) found that approximately $5 \%$ of deaf children in developing countries had no access to any education. The survey also found that 19 of the countries represented in the study did not recognise deaf persons as equal to other citizens in their countries and only 44 states had legal recognition of sign language. The same studies also indicated that the deaf worldwide look at themselves as a linguistic, cultural, and social community. The history of the deaf is to a far extent very much linked with that of sign language and also is an integral part of what is called deaf culture, a culture to be understood in a collective sense, corresponding to a benchmark of values linked to a shared history. Banning the use of the SL during a given period was long felt by the deaf as oppression or as linguistic imperialism to use Ladd's words. Deaf people use the sign and the written word abuse, which suggests the violence felt and expressed by the deaf community, echoing its history and its relationship to the hearing community.

As with all linguistic minorities, there is usually a significant amount of diversity among members of this community; but there is one main characteristic for all. Many individuals with hearing loss see themselves as disabled and therefore call themselves "hearing impaired". Often these people have lost their hearing after learning the spoken language, so it is understandable that they see themselves as disabled in the sense that they have lost the ability to hear their first language directly. Others were born without hearing or lost in their early childhood; some were born to hearing parents. Life without the sense of hearing is a normal life where he/she does not feel like losing anything. Such individuals proudly refer to themselves as "deaf" and can be considered a cultural and linguistic minority (Paden, 1980). This fundamental difference between the two groups has wide-ranging implications regarding language selection and services provided by the interpreter.

On the contrary, many of the individuals who suffer from hearing impairment do not use sign language, but they have learned instead to use a variety of languages to interact with the public. People who wish to refer to other types of signed languages also need translation services in general, but because it offers these services to convey words on a one-to-one basis. The situation is similar in other countries; many have their sign language with their own grammatical structures, and their system to communicate the vocabulary of the spoken majority languages. Thus, the interpreter who works with the deaf knows at least how to be a vector of words. 


\section{Sign Language (SL)}

$\mathrm{SL}$ is the language of the deaf community in face-to-face communication, learned as the mother language or as the preferred language for the deaf. Sign language uses hand movements, facial expressions, and body language to communicate. Wilcox, Perrin, and Jarque (2003) indicated that:

Signed languages are natural human languages used by deaf people throughout the world as their native or primary language. Although there has been no formal survey of the world's signed languages, linguists assume that they number in the hundreds. (p. 139)

Sign language is a product of human creative activity. It can present new cultures, relay cultural values, be a way of exchanging experiences, as well as be a source of inspiration for cultural transformations. Also, a sign language is an example of a remarkable and delicate cultural artefact worthy of consideration and safeguarding. It is the deaf person's contribution to overall human culture (Woll \& Sutton-Spencer, 2007). In her study on the history of American Sign Language (ASL), Wilcox et al. (2003) wrote that:

Like spoken languages, signed languages may be classified into genetic groups. These genetic relations follow the historical development of signed languages, and so do not reflect the same relations as may exist for spoken languages in the same areas. For example, French Sign Language (LSF) is a parent language of both American Sign Language (ASL) and Russian Sign Language. British Sign Language appears to be genetically unrelated to French Sign Language. (p. 140)

Furthermore, the deaf who were born deaf consider it as their native language, and like many languages, it has its grammatical structure: The verb does not express time and does not have a formula for the unknown, a language characterised by many interpretations and the relationship between the actor, and the object is not reflected in the order of speech (Baker \& Cokely, 1980). Wilcox et al. (2003) indicated:

The gestural-visual nature of sign languages is mirrored in their linguistic structure. Signed languages make extensive use of space, for example by incorporating spatial locations to indicate verbal arguments; in addition to the hands, the face plays a critical role in signed language grammar, expressing a range of information such as questions, topic, adverbials, and so forth. American Sign Language (ASL), like many signed languages, is highly synthetic with tendencies towards poly-synthesis. ASL allows morphemes indicating action, person agreement, aspect, and adverbial information to be combined into a single, multimorphemic ASL word; for example, "I very carefully gave [one] to each [person]" would be expressed with a single sign in ASL. (p. 126)

Thus, the interpreter of the deaf deals with two different natural languages, each with a different composition, such as the difference between English and French. A growing number of American universities have recognised and accepted the American designation of deaf as a foreign language in their system of documenting foreign languages. Aarons (1994) in her study indicated that:

One strongly-held belief was that ASL sentence structure is determined by the discourse organisation of ASL, which follows a topic-comment style of speech presentation. Friedman (1975) claimed that ASL had no strict word order rules and that ASL sentence structure itself was ordered regarding the discourse principle of topic-comment: the introduction of the topic, followed by the comment; and that grammatical relation, such as subject, verb, and object were not relevant to ASL sentence structure. Many other researchers did not adopt such extreme views about how ASL is different from English, but there was a strong emphasis on showing that ASL behaved very differently from English. Thus, the aspects of ASL that were highlighted were those features that are most strikingly not like English: the variable word order, the classifier system of ASL, non-manual grammatical marking, and crucially, the use of space in ASL. (p. 26) 
Historically, it is worth saying that the oldest attempt to develop education for the deaf started in 1555 by the Spanish (Pedro Ponce). He began educating the children of noble families, followed by another Spanish called Juan Pablo Bonet who in 1620 published the first book about deaf education and invented the first finger spelling, where he developed the early known pictograms of sign language hand shape. A hundred years later, the French L'Abbée de L'Epée (1712-1789) is one of the world-renowned figures for his work and his involvement in sign language, in a context where deaf people were not socially considered. He is still regarded as the father of deaf education since he established the first school for the deaf in Paris in 1771. His writings enabled many people to situate the beginning of his teaching with young deaf people towards the second part of the 18th century (around 1760). Achievements of deaf students were spread all over Europe. This period in France, is known as the "golden age" for sign language for about a hundred years. France highly innovative educational practices were heard abroad, and French deaf teachers who became directors of more than 70 schools were solicited everywhere especially in America, where the first school for the deaf using sign language was set up in 1817 in Hartford, Connecticut. It was called The American Asylum for the deaf by Thomas Hopkins Gallaudet, who made a trip to Europe to learn more about the methods of teaching deaf students and training teachers. He aimed at bringing back a framework for instructing hard of hearing kids to the USA. In France, he met the Abbé de L'Epée, who had built up a teaching system for educating deaf children to read and write. He initiated the use of "methodical signs". It is often considered by researchers in deaf studies to be one of the early initiatives to "systematise" the aboriginal dialects being used by hard of hearing individuals in France. Gallaudet managed to convince Laurent Clerc, one of the best deaf instructors in France at that time, to accompany him to America; Laurent Clerc became the principal educator of the deaf at the leading school in the United States.

In the 19th century, the extraordinary congress in Milan was held in 1880, with the aim of bringing together all the European specialists in the education of young to decide on a single method of teaching. The congress unanimously voted, without appeal for SL supporters, that SL should be banned because of its supposedly pernicious influence on learning the oral language. Proponents of the oral method which were head by the famous Graham Bell excluded sign language entirely from schools for the deaf in the world and imposed the oral method. The latter remained the primary accepted method in Europe and America, but the power of sign language and its importance never disappeared from the spirit of the deaf in America Who set up the National Association which is considered as the first protest movement against the Milan Congress. This action helped to keep sign language alive in America taking the form of deaf clubs and community celebrations and gathering in different cities. This allowed SL to develop and take its place in the deaf community until the adoption at a conference, World Federation of the Deaf held in Japan in 1991 of a resolution stipulating that sign language is the language of the deaf.

In 1955, William Stoke joined the teaching staff at the University of Gallaudet in Washington, D.C., the only university in the world specialised in the education of deaf and hard of hearing students. As an assistant professor in the Department of English, like other faculty members, he had no experience or knowledge of American Sign Language. At the time, there were no classes to teach American Sign Language as a language with its own rules and structures. They considered American Sign Language to be inadequate and not reach the level of living human languages and were merely references to facilitate communication with the deaf but did not live up to the real language. Armstrong and Karchmer (2002) wrote: 
Sign Language is equated with the despised, non-alphabetic writing system of a non-western people (the Chinese), it is said to lack the precision of speech, and it is stated, without any evidence, that deaf people will not achieve their full potential through its use. When Stokoe arrived at Gallaudet in 1955, he was entering an environment that was dominated by thinking like this. His achievements on the value of signed languages were essentially fourfold. Stokoe's first achievement was to realise that the Sign Language his students used among themselves had all the essential characteristics common to spoken languages and that it had the same potential for human communication. His second achievement was to devise a descriptive system that would convince language scholars of these facts. (p. xii)

Students at Gallaudet were studying some indicative vocabulary only. When Stokoe joined these classes to learn SL vocabulary for three weeks, he ended up the course by teaching deaf students, and communication with them was done through speaking English with some signs at the same time. This method was known as Simultaneous Communication or SimCom. No one dared believe that the use of such method (simultaneous communication) would help deaf students to acquire English. Stokoe (1960) described this situation as follows:

I have known that deaf people when they are together and communicate with each other among them, that what they communicate with is a "real language", and since the language they communicate with is not English, they must have their language, there are no so-called broken or inappropriate words, they communicate in a pleasant and smooth way. (p. 345)

In 1957, Stokoe began acute experiments on American Sign Language as he was entirely convinced that it was a complete and independent language with the characteristics and qualities of living human languages. After three years of continuous research, Stokoe published his research: Sign Language Structure: "An Outline of the Visual Communication System of the American Deaf". This research was the first linguistic analysis of a signed language in which, William Stoke showed that ASL and all the signed languages used by deaf people in all countries of the world are real human languages with correct living rules and structures that differ from the standards and compositions of spoken languages. The reactions of deaf students and hearing faculty members to the results of his research were so fast and powerful. Despite all the attack he received, Stokoe continued his research till when in 1965, he together with Dorothy Casterline and Carl Croneberg published The Dictionary of American Sign Language on Linguistic Principles. In 1970, many linguists and psychologists began studying the characteristics of ASL. At the time, their published research usually started with a brief explanation of why ASL was a language. Such comments were necessary as many people still believed that sign language was not a language. Studies in various grammatical aspects have proved beyond a shadow of a doubt that ASL is a real human language. He often called for more diffusion of sign language that the more it is used, the more it would spread. It would also develop similar to the development of spoken language regarding changing dialects and their diversity in the same way through which languages, such as French, Spanish, Italian, and other romantic languages developed gradually and evolved from Latin. His arguments were because when a large number of people use a language, it may contain a significant amount of variation and diversity and this can be seen in ASL used by more than half a million-deaf people. Some of these differences are formerly regional, but others are due to the age at which sign language has been learned, as well as social factors, such as the home environment (hearing or deaf parents) and the educational background of the person using the language.

Many deaf people use other forms of signing called PSE (Pidgin Signed English). Primarily, this consists of using ASL signs in English-like word order. Communication of this kind was actively fortified in some schools for the deaf. Afterwards, the invention of manual codes for English (Signed Exact English) was forced as the 
"language" of communication in many schools. Also, deaf people often switch to an English-like order when signing to hearing people. Spoken languages differ in rules and structures, as well as the signed languages that differ in rules and structures. For example, the Arabic language differs in its rules from English; sign language differs regarding grammar and syntax of another language. One more issue is that sign languages are never associated with spoken languages, and the fact that two or more states use one spoken language does not mean that they are using one sign language. For example, the United States, Britain, and Australia speak one spoken language, English, but these countries use differentiated languages different from each other. ASL is very distinct from the British Sign Language, and each of these two languages has different rules and structures.

\section{Misconceptions About Sign Language}

One of the misconceptions about the languages that indicate that sign language is a literal translation of the spoken language. For example, many people believe that a sentence or text in a spoken language can be translated literally with the same rules and syntax in Arabic and they forget that the sign language has syntax and rules that are entirely different from those of Arabic. For example, when translating an English sentence into Arabic, we do not adhere to the same order, syntax, and rules of English. We read the sentence and convert the meaning with the rules and syntax of the Arabic language (the target language).

\footnotetext{
A common misunderstanding is that signed languages are merely representations of spoken languages-that ASL, for example, is a signed representation of spoken English. Signed languages, however, are independent languages with their own lexicons and grammars. Like spoken languages, signed languages have genetic and historical relations with other signed languages. ASL's closest genetic relative, for example, is French Sign Language (LSF). (Gonzalez-Marquez \& Irene Mittelberg, 2006, p. 171)
}

Besides, it has been argued that sign language is deficient in comparison to spoken languages because it does not have a varied vocabulary like spoken languages nor the number of grammatical forms. Another common false idea is that signed languages merely (or primarily) make excessive use of finger-spelling and which is not true because fingerspelling comprises only 26 handshape configurations that correspond to the alphabet. It is mainly used to designate technical terms and proper nouns. Another widespread misconception also is that sign language is not a real proper language, and it is only a sophisticated system of gestures. Thus, it is very iconic by nature because all the signs that are used by the deaf community in one country are not genuinely arbitrary, but that there is a relationship between the sign and what it stands for. Some people see that signed languages have no rules and structures but are attempting to communicate through the gestures. Many people believe that the languages are uniform in all countries of the world, while in fact, they differ from one country to another, but from one region to another precisely as the difference between languages spoken in countries and the different dialects between regions.

There may be more than one language in a country, for example, in Canada where there are two distinct languages: American Sign Language, which is used in most regions of Canada, and the French Sign Language used in Quebec. The French Sign Language used in the territory of Quebec differs from the French Sign Language used in France and Finland has three sign languages. There are other misconceptions about sign languages, but which have been proven untrue by research conducted in various parts of the world mainly at the level of Gallaudet University. 


\section{The Sign Language Interpreter (SLI)}

A sign language interpreter is an expert responsible for interpreting everything that is said or signed. He also delivers sign language interpreting and other tactual and visual communication forms, transliteration, and translation services to deaf, hard-of-hearing, and deaf-blind individuals. Interpreters attempt to remain impartial toward the content they work on and try not to amend or modify the sense or tone of what is conveyed. His job also consists of rendering the hearing speaker's terms into the sign language used by the deaf party. When deaf person signs, an interpreter will convert the sense signed into the spoken language for the hearing interlocutor, which is sometimes referred to as voice interpreting or voicing. Historically, interpreting for the deaf has been mentioned in different documents for centuries. The need for sign language communication mediators between hearing and deaf people has been since the first oldest mentioned times in 427-347 BC (Per Eriksson, The History of Deaf People). From 1928 onwards, we begin to have both deaf and hearing people qualifying under as welfare workers with this qualification, which included interpreting alongside other skills tested, and interpreting was an expectation of the welfare workers role until the mid-1970s (Stone, Walker, \& Parsons, 2007).

By the 18th century, we start to have accounts of Deaf people within the legal system (Stone and Woll, 2008) and then in 1817 in Glasgow, a "Deaf aide" was used in the trial of Jean Campbell, when the headmaster of Edinburgh Institution for the Deaf and Dumb, Robert Kinniburgh, was unable to understand and be understood (Hay, 2008). From 1928 onwards we begin to have both Deaf and hearing people qualifying under as welfare workers with this qualification, which included interpreting alongside other skills tested, and interpreting was an expectation of the welfare workers role until the mid-1970s. (Stone et al., 2007, p. 2)

Moreover, transliteration seems to be the purest form of expression for a hearing person who has not learned to integrate the multidimensional aspect of the sign language nor understood the importance of it. Moreover, the extended use of sign language seems to be commonly accepted as a stage allowing the acquisition of space (which we can relate to grammar), but in no case, can language be limited to this step alone. It is very common to observe that many curricula welcome students to LS interpretation without any prior knowledge of the signed language. Without sufficient linguistic immersion or language proficiency, these future interpreters are thus confronted with their linguistic limits, which despite themselves restrict them to transliteration alone. Winston defined transliteration as a specific form of sign language interpreting. It is the process of changing one form of a message, either in spoken English or French or Arabic English, French or Arabic into the other signed form of these languages. Winston elucidates that:

Transliteration: (transliterating) is a specific form of sign language interpreting. It is the process of changing one form of an English message, either spoken English or signed English, into the other form. The assumption in transliteration is that both the spoken and the signed forms correspond to English, the spoken form following the rules of standard English and the signed form being a simple recording of the spoken form into the manual code of expression. (Winston, 1989, p. 147)

There are different specialised interpreting situations in which a SLI may be operating:

- Legal interpreting: In settings, such as court actions lawyer's consultations.

- Medical and mental health interpreting: In medical settings, such as doctor's appointments, inpatient hospital procedures, or consultations, 
- Educational interpreting may be performed in an educational setting during classes, such as primary or secondary schools, and colleges and graduate schools.

- Spiritual interpreting takes place in locations that are in religious nature.

- Deaf-blind interpreting occurs at close visual range mainly using sign language and fingerspelling deaf-blind sign language received by a sense of touch with one or two hands (tactile), and close-range sign supported speech.

- Oral interpreting: Oral transliterators (also called voice interpreters). The oral interpreter silently mouths/whispers sentences to the deaf or hard of hearing the person, changing words or phrases as needed, to ones that are easier to speech-read. They may also "voice" for speakers who are voiceless, or whose voices are difficult to understand. The interpreter enunciates, repeats, or rephrases a speaker's remarks using natural lip movements and gestures.

- Cued speech translators (coders) translate the spoken message into a cued spoken language.

- Live captioning: offering live captioning services, which may be distant on onsite to clients who are deaf or hard of hearing for a variety of settings including courts, conferences, educational settings, and workplace meetings. It can be used on different devices, such as TV screens, tablets and smartphones, laptops, and white screens.

- Notetaking consists of taking notes at events and gatherings, to record what is taking place for a deaf or hard of hearing person.

Sign language interpreters, however, may have some differences that appear in daily practice and should be within the existing deaf audience's vision and therefore stands next to the native speaker instead of sitting in a cabin. Forestal (2005) explained:

Deaf Interpreters now practice in multiple settings, such as courts, hospitals, work-related sites, training programs, conferences, theatres, and classrooms across the country, primarily in major cities. They work as translators from spoken or written English into ASL, international sign language, or in a gestural form. They are now everywhere in the field of interpreting with Deaf people and where ASL-English interpreting occurs. (p. 235)

In such circumstances, an interpreter may interact with the deaf to clarify or request some moments during which he/she can convey a specific complex message. Moreover, because deaf communities meet people who speak a different language on a daily basis, the interpreter who works in this community gets regularly updated within these communities since he/she is considered as entirely belonging to it.

Upon initiating the interaction, the deaf person and the interpreter chat briefly. The nature of the gaze during this conversational interaction is not much different from what might be experienced in an interaction between hearing people using spoken language who are first meeting one another; eye contact is direct and sustained. Once the actual task of interpreting begins, a shift in gaze takes place. The deaf participant using interpreting services is dependent on the interpreter for access to communication; in a visual language, this means that eye contact must be sustained over time. If the eye contact or gaze is broken, vital information may be lost. It is not just a deaf person who must maintain this eye contact; all parties (whether deaf or hearing) who use a signed language must engage in the gaze. Contrast this with a spoken- language conversation between two relative strangers, where eye contact is much reduced. (Dirksen \& Bauman, 2014, p. 11)

Furthermore, SLIs interpret into a variety of contexts ranging from doctors' appointments to classes, wedding parties, marital counselling sessions, work interviews, and even psychotherapy. That is why Smith 
(1983) emphasised that sign language interpreters "must not only translate the mind and ideas but the heart and spirit" of a speaker. Another difference is that the sign language interpreter may receive training on one or both assignments, namely interpreting and transcription. This distinction can be understood only in deaf communities. The situation in the United States is a model for that process, but there are key points that apply equally to most developing countries.

These unique characteristics are what make the job of SLIs both fantastic and so unique. A sign language interpreter should never have moustaches, a beard, or circle beard. He must try to speak directly to the deaf to maintain communication because the deaf may request clarification from the interpreter to ensure the accuracy of the information conveyed. Besides, he may be forced to fingerspell or write technical terms or jargon relating to a particular field or concept, either on the chalkboard, an overhead projector, a class handout, or with some other visual aid. People should speak at a reasonable pace because interpreters frequently start interpreting with a time lag of more than two sentences after the voices lecturer since interpreters need first to grasp the meaning of the information and then relay it. Speakers need to speak with natural and modest speed, taking into consideration that the SL interpreter must listen, process, and understand a complete thought before signing it.

The relevant use of space makes it possible to set up entities in the area of signing and then create interactions between these spaces that will allow the speaker to create meaning. Space represents in LS somehow what grammar is to French. It will be in the space of signing that time will be introduced, that all forms of discursive processes may take shape and that discourse will evolve according to the arrangement proposed by the speaker. This is what researchers call "spatial mapping" in sign language: Spatial mapping is "usually defined as the use of space for locating referents to create a "picture" of some physical, real-world event. This is one type of spatial mapping, and it is an essential feature in SL. However, it is only one form of the much larger feature of spatial mapping in SL (Baker \& Cokely, 1980; Klima \& Bellugi, 1979). Spatial mapping includes not only the "drawing" of a picture, but it also includes the space to build relationships between abstract ideas. It also means to compare entities, to add imagery and detail, to describe both physical attributes, such as color, size, and shape as well as non-physical attributes, such as attitudes, emotions, and beliefs, to show the passage of time, and to contribute to the prosody of ASL (Winston, 1996).

This definition is interesting since Winston presents a set-based view of the physical framework of the space of signing with what this space allows regarding dynamic content generating meaning. The researcher gives importance to a "relevant use of space", for example, by the tactic of imagining a scenario because it is much more than the mere imagery. Winston's contribution is also interesting from the translational point of view since she takes up the problem of training in interpretation alongside the linguistic analysis of this tactic.

Besides, all the communications should be in turn because the deaf will not follow up with the discussion in there is more than one person is talking at a time. The interpreter also needs to allow enough time for the deaf to read and to follow overhead projections/multimedia presentations before signing. On the other hand, note taking is required when lipreading is used because the interpreters will need to spell out the words in such a way that the deaf person understands. It is hard to take good notes while watching a sign language interpreter or reading on the lips. 


\section{The Occupation of a SLI}

The position of the sign language interpreter varies from country to country. In the 1960s, education programmes for deaf children began to accept sign language as a guiding medium in many countries. This, along with other factors, led to an organised effort to provide professional sign interpreters to the community. For example, in the United States, the Register of Interpreters of the Deaf(RID) was established in 1964, representing professional interpreters, and in 1994, it had about 5,000 members, of whom 2,400 were accredited. To obtain a certificate, an individual must first pass a written test covering a range of ethics and community-related knowledge and testing the knowledge of the deaf culture. Passing the written test makes one eligible to continue one or both certificates either by passing its own or a performance test. Sweden, for example, has a long history of respect for the language and culture of the deaf and has a well-organised group of interpreters. Other countries, such as Switzerland are still on the way of organising the profession. In Canada, there is a professional organisation, the Association of Visual Language Interpreters in Canada (AVLIC), which was established in 1979. The organisation has nine affiliated bodies throughout the country (Russell, 1994), followed by about 400 members. The UK, such as the Association of Sign Language Interpreters (ASLI), represents the interpreters in England, Wales, and Northern Ireland, while the Development Board for Deaf Communication is administered by the Scottish Community of Sign Language Interpreters (SASLI). Regarding other countries in Europe, Denmark, Ireland, the Netherlands, Sweden, Germany, and Belgium, Switzerland and Finland established in 1993 the European Forum of Sign Language Interpreters (EFSLI). The main aim was behind the creation of this organisation is because the training level received by the sign language interpreter is far behind the degree of training received by the spoken language interpreters.

For sign language interpreters in the United States; however, the clear majority of them continue for only two years, after which an individual can enrol immediately after high school. Because the American Sign Language and other sign languages are relatively rare as a second language, the education programs do not require the applicant to be familiar with any of them. Students receive instruction in the language during the program. Often this was limited to learning vocabulary followed by learning to transcribe. However, there is an increasing tendency to award higher degrees in the field of Sign Interpreting. At the end of 1994, there were fewer than 10 four-year university-level training programs in the United States; and only one at Gallaudet University. Training in these programs included interpreting between English and ASL; parallel teaching methods used with interpreters of spoken languages should start interpreting first before interpreting begins, although no notes are recorded in the consecutive interpreting, and therefore the paragraphs are shorter. The nature of the interpreting between spoken languages and sign languages is a language learning challenge. It becomes complicated for students, and there is no particular geographic location where ASL or any other sign language is the language of the majority, so full integration is impossible.

\section{Conclusion}

The emphasis on quantity more than quality meant that the clear majority of interpreters today had very little or no relationship with the deaf community before they were trained. Many of them knew only one spoken language but not the sign of the deaf. In the past, translation services were provided by those who were linked to deafness from early childhood; and since they have close ties to the deaf community. The number of interpreters 
today is vastly higher than before; this may lead to better service for a community with hearing disabilities; however, it often finds it very difficult to obtain an interpreter who can understand and produce their native language. The deaf always find themselves in unusual situations, and they are forced to adapt themselves with the interpreter and not vice versa. This shift from a member of the community to a neutral professional person-and vice versa-was reflected in an equal change in the way the interpreter's role is perceived. Many of the titles that have likened these interpreters to "assistant", "intervener", and "mediator" have appeared in attempts to find a suitable name for their job. One of the last names they were called was "agent", "cultural mediator", and "ally". These words reflect the belief that interpreters working with the deaf should be involved in the empowerment process.

\section{References}

Aarons, D. (1994). Aspects of Syntax of ASL (Ph. D. thesis, Boston University).

Armstrong, D. F., \& Karchmer, M. A. (2002). The study of signed languages. Washington, D.C: Gallaudet University Press.

Baker, C., \& Cokely, D. (1980). American Sign Language: A teacher's resource text on grammar and culture, Silver Spring Publishers.

Bauman, H. L. (Eds.). (2008). Introduction: Listening to deaf studies. In Open your eyes: Deaf studies talking (pp. 1-34). Minneapolis: University of Minnesota Press.

Cerney, B. E. (2004). Relayed interpretation from English to American Sign Language via a hearing and deaf interpreter (Unpublished doctoral dissertation, Union Institute, Cincinnati, OH).

Dirksen, L., \& Bauman, J. J. M. (2014). Deaf gain. London: University of Minnesota press Open.

Forestal, E. (2006). Effective practices for teaching deaf interpreting processes. In E. M. Maroney (Ed.), Proceedings of the 16th National Convention Conference of Interpreter Trainers (pp. 11-18), Monmouth, OR: Western Oregon University.

Forestal, E. (2005). The emerging professionals: Deaf interpreters, their views and experiences in training. In M. Marschark, R. Peterson, and E. Winston (Eds.), Sign language interpreting and interpreter education (pp. 208-234). New York, N.Y.: Oxford University Press.

Isham, W., \& Lane, H. (1993). Simultaneous Interpretation and the recall of source language sentences. Language and Cognitive Processes, 8(3), pp. 241-264.

Klima, E., \& Bellugi, U. (1979). The signs of Language. Cambridge: Harvard University Press.

Langholtz, D. (2004). Deaf interpreters today: A growing profession. WFD News, 17(1), 17. Helsinki, Finland: World Federation of the Deaf.

Gonzalez-Marquez, M., \& Irene Mittelberg, M. J. S. (Eds.). (2006). Methods in cognitive linguistics. Amsterdam: John Benjamins Publishing Company.

Padden, C. (1980). The deaf community and the culture of deaf people. In Baker, C \& R. Battison (Eds), Sign language and the deaf community. Silver Spring, MD: National Association of the Deaf.

Padden, C., \& Humphries, T. (2005). Inside deaf culture. Cambridge, MA: Harvard University Press.

Registry of Interpreters for the Deaf Professional Standards Committee. (1997). Standard practice paper: Use of a certified deaf interpreter. Retrieved April 28, 2007, from http://www.rid.org/UserFiles/File/pdfs/120.pdf

Ressler, C. (1998). A comparative analysis of a direct interpretation and an intermediary interpretation in American Sign Language (Unpublished master's thesis, Gallaudet University, Washington, DC).

Rusell, C. (1994). Sign language interpreting in Canada. Paper presented to the third National Congress of the Canadian Translators Interpreters Council. Banff, Alberta. Canada.

Smith, T. (1983). What goes around comes around: Reciprocity and Interpreters. The reflector 5, (Winter), 5-7.

Stokoe, W. C. (1960). Sign language structure. Silver Spring, MD: Linstok Press.

Stone, C., Walker, J., \& Parsons, P. (2007). Professional recognition for deaf interpreters in the UK. Retrieved from http://discovery.ucl.ac.uk/1318640/1/1318640_Stone_Walker_Parsons_Sept_2011revised.pdf

Wilcox, S., Perrin, P., \& Jarque, M. J. (2003). Mappings in conceptual space: Metonymy, metaphor, and iconicity in two signed languages. Jezikoslovlje, 4(1), 139-156.

Winston, E. A. (1996). Spatial mapping in ASL discourse. Printed in CIT Proceedings—Assessing Our Work: Assessing Our Worth, 11th National Convention, Little Rock, Arkansas. 
Winston, E. A. (1993). Spatial mapping in comparative discourse frames in an American Sign Language lecture (Ph.D. dissertation, Georgetown University, Washington, DC.

Winston, E. A. (1989). Transliteration: What's the message? In C. Lucas (Ed.), The sociolinguistics of the deaf community (pp. 147-164). Washington, D.C: Gallaudet University Press.

Woll, B., \& Sutton-SpenceR. (2007). Sign languages: Cambridge language surveys. Cambridge: Cambridge University Press. Retrieved from http://discovery.ucl.ac.uk/123958/ 